\title{
Correction to: Romosozumab: A Review in Postmenopausal Osteoporosis
}

\author{
Julia Paik ${ }^{1}$. Lesley J. Scott ${ }^{1}$
}

Published online: 21 October 2020

(c) Springer Nature Switzerland AG 2020

\section{Correction to: Drugs \& Aging https://doi.org/10.1007/s40266-020-00793-8}

Unfortunately the following sections were published incorrectly in the original article.

\section{Section 2: Pharmacodynamics}

In Paragraph 2, the following sentence which previously read:

"In FRAME [11] and ARCH subgroup analyses $(n=266$ in ARCH [12]) and in the STRUCTURE [13] study, P1NP levels peaked in the first month with romosozumab before falling below baseline levels after 6-12 months; at months 1 and 12 (and at month 13 in FRAME [11]), P1NP levels in the romosozumab group were significantly increased compared with baseline levels $(p=0.006)[11,13]$ and with other treatments (alendronate, an antiresorptive agent [12] and teriparatide [13]; $p=0.001$ for both comparisons)."

Should read:

"In FRAME [11] and ARCH substudies ( $n=266$ in ARCH [12]) and in the STRUCTURE [13] study, P1NP levels peaked in the first month with romosozumab before falling closer to [13] or below [11,12] baseline levels after 6-12 months. At month 12 (after which point patients switched treatments; see Sect. 4), P1NP levels were significantly reduced in patients who had received romosozumab compared with baseline levels $(p \leq 0.006)[11,13]$ and

The original article can be found online at https://doi.org/10.1007/ s40266-020-00793-8.

Julia Paik

demail@springer.com

1 Springer Nature, Private Bag 65901, Mairangi Bay, Auckland 0754, New Zealand teriparatide in STRUCTURE ( $p \leq 0.001)$ [13], but higher than with alendronate in $\mathrm{ARCH}(p<0.001)[12] . "$

\section{Section 4: Therapeutic Efficacy}

In Paragraph 1, the following sentence which previously read:

"The efficacy of subcutaneous romosozumab $210 \mathrm{mg}$ in ambulatory postmenopausal women with osteoporosis was investigated in the 24-month [11] or $\geq 24$-month [12], double-blind, multinational, pivotal, phase III FRAME $(n=7180)$ [11] and ( $n=4093)$ [12] trials, and the 12-month, open-label, multinational phase IIIb STRUCTURE trial $(n=436)$ [13]."

Should read:

"The efficacy of subcutaneous romosozumab $210 \mathrm{mg}$ in ambulatory postmenopausal women with osteoporosis was investigated in the 24-month [11] or $\geq 24$-month [12], double-blind, multinational, pivotal, phase III FRAME $(n=7180)$ [11] and ARCH $(n=4093)$ [12] trials, and the 12-month, open-label, multinational phase IIIb STRUCTU RE trial $(n=436)$ [13]."

\section{Section 4.1: Incidence of Fractures}

In Paragraph 2, the following sentence which previously read:

"After 24 months in FRAME, this risk was reduced by $33 \%$ in the romosozumab-to-denosumab group relative to the denosumab-only group (Table 1); this endpoint was considered to be exploratory due to previous endpoints in the testing sequence (cumulative incidences of nonvertebral fracture after 12 and 24 months) not reaching statistical significance [11]." 
Should read:

"After 24 months in FRAME, this risk was reduced by 33\% in the romosozumab-to-denosumab group relative to the placebo-to-denosumab group (Table 1); this endpoint was considered to be exploratory due to previous endpoints in the testing sequence (cumulative incidences of nonvertebral fracture after 12 and 24 months) not reaching statistical significance [11]."

\section{Section 6: Place of Romosozumab in the Management of Postmenopausal Osteoporosis}

In Paragraph 2, the following sentences which previously read:

"Of note, while the risk of nonvertebral fracture was not significantly improved after 12 months' treatment with romosozumab, it appeared to improve 1-2 years after patients had switched to another anti-osteoporotic treatment (Sect. 4.1). Further studies may help in better determining whether this delay in benefit is directly related to romosozumab treatment."

Should read:

"Of note, while the risk of nonvertebral fracture was not significantly improved after 12 months' treatment with romosozumab (potentially reflecting a lack of statistical power), it appeared to improve 1-2 years after patients had switched to another anti-osteoporotic treatment (Sect. 4.1)."

The Original article has been corrected. 